\title{
Soziale Arbeit in Betrieben
}

\section{Ergebnisse einer Studie zu Qualifikationen und Aufgaben in der betrieblichen Sozialarbeit}

Die betriebliche Sozialarbeit (BSA) blickt in Deutschland auf eine wechselhafte und unstete Geschichte zurück und könnte sich u. a. im Zuge veränderter psychischer Anforderungen in der Arbeitswelt zu einem wichtigen Tätigkeitsfeld entwickeln. Allerdings ist wenig über diejenigen bekannt, die in dem Feld tätig sind. Wir präsentieren in diesem Beitrag Ergebnisse einer empirischen Studie zu der Frage, welche Qualifikationen und Aufgaben das Personal in der BSA hat.

$\mathrm{I}$ $\mathrm{m}$ Zuge veränderter psychischer Anforderungen an Beschäftigte hat das BMAS-geförderte Projekt „Psychische Gesundheit in der Arbeitswelt" einen Leitfaden veröffentlicht, damit Betriebe einen Zugang zum Thema Mitarbeiterberatung finden (vgl. psyGA 2017). Obwohl die betriebliche soziale Beratung von Beschäftigten in Deutschland auf eine lange und wechselvolle Geschichte zurückblicken kann (vgl. u. a. Blandow 1993), ist wenig über sie bekannt, so z. B. über die Zahl der Personen, die in der betrieblichen Sozialarbeit tätig sind. Bremmer (2017a) zufolge sind etwa 300 Personen im Bundesfachverband organisiert, welche wiederum in 150 Unternehmen arbeiten. Trotzdem ist von einer hö-

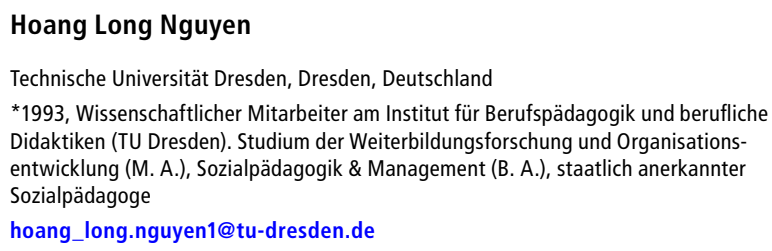

\section{Sandra Bohlinger}

Dresden, Deutschland

*1973, Dr. phil. habil., Professorin für Erwachsenbildung mit den Schwerpunkten berufliche Aus- und Weiterbildung sowie komparative Bildungsforschung am Institut für Berufspädagogik und berufliche Didaktiken (TU Dresden)

sandra.bohlinger@tu-dresden.de

Zusammenfassung Betriebliche Sozialarbeit ist ein in der Forschung und Praxis wenig wahrgenommenes berufliches Tätigkeitsfeld der Sozialen Arbeit. Folglich gibt es kaum Forschungsbefunde, die sich mit dem Personal in diesem Feld beschäftigen. Der Beitrag präsentiert zentrale Ergebnisse einer qualitativen Studie in der betrieblichen Sozialarbeit. Dazu wurden Stellenausschreibungen analysiert und betriebliche Sozialberater/innen interviewt. Der Beitrag zeigt, welche Qualifikationen benötigt und welche Aufgaben wahrgenommen werden.

Schlüsselwörter Betriebliche Sozialarbeit, Berufsbild, Qualifikation, Arbeitsaufgaben heren Zahl an betrieblichen Sozialarbeiter_innen (BSAMA) auszugehen, da viele in dem Feld Tätige Aufgaben der betrieblichen Sozialarbeit nur als Teil ihrer Tätigkeit ausführen dürften.

Darüber hinaus gibt es ein breites Spektrum an Bezeichnungen für jene Einheiten, in denen BSA angeboten wird. Mit Rekurs auf das Mitgliederverzeichnis des Bundesfachverbandes listet Bremmer (2017a) u. a. auf: Beratungsdienst für Mitarbeiter_innen, Anlaufstelle für soziale Angelegenheiten, Betriebsbeauftragter für Sozialbetreuung, psychosozialer Dienst für die Beschäftigten, Mitarbeiterberatung, betriebliche Suchtberatung. Dies deutet auf eine starke Binnendifferenzierung im Berufsfeld hin, die mit unterschiedlichen Zugängen, Aufgabenprofilen und Handlungsfeldern einhergeht. Klein und Appelt (2017) beklagen „beträchtliche Informationsdefizite“ zu den Anforderungen, Strukturen, Arbeitsinhalten und Arbeitsmethoden. Andere Autoren bezeichnen die Forschung zur BSA als „rudimentär“, denn über „die Verbreitung, die Aufgabenprofile von Betrieblichen Sozialberatungen, ihre Organisationsformen, die Qualifikationen von Sozialberatenden im Feld oder die bearbeiteten Problemlagen der Klientel ist kaum empirisches Wissen vorhanden" (Baumgartner und Sommerfeld 2016, S. 27). Umso mehr scheint es bedeutsam, sich mit dem Personal in dem Feld auseinanderzusetzen. Wir ergänzen dieses fragmentarische Bild um einen Beitrag zum Berufsbild von BSA-MA, indem wir uns im Rahmen einer qualitativen Untersuchung an der Schnittstelle zwischen Sozialer Arbeit und Berufswissenschaft mit zwei zentralen Fragen beschäftigen: Welche Qualifikationen werden in der BSA benötigt? Welche Aufgaben werden in der BSA ausgeführt?

\section{Methodisches Vorgehen}

Zunächst haben wir in einer Dokumentenanalyse (vgl. Mayring 2016) 15 Stellenanzeigen im Bereich der BSA ausgewertet. Auf Basis der Ergebnisse wurde ein Leitfaden für ein problemzentriertes Interview nach Witzel 
(1985) konzipiert, welcher in einem Pre-Test erprobt wurde. Die Triangulation zweier qualitativer Erhebungsmethoden sollte dazu beitragen, möglichst vielfältige Daten zu erhalten und verschiedene Perspektiven zu berücksichtigen (vgl. Flick 2008). Insgesamt wurden im Zeitraum von Februar bis Juli 2018 sieben Interviews mit in dem Feld tätigen Personen (zwei von ihnen in Führungspositionen) geführt. Alle Interviews wurden wörtlich transkribiert und mittels der strukturierenden Inhaltsanalyse ausgewertet (vgl. Mayring 2016). Wohl wissend um die begrenzte Aussagekraft der Datengrundlagen liefern die Ergebnisse einen Einblick in ein heterogenes und schwer überschaubares Feld. Anders als bisherige Studien zu Anforderungen und Aufgaben in der BSA (v. a. Baumgartner und Sommerfeld 2016; Stoll 2013) nutzen wir einen qualitativen Forschungszugang, um detaillierter Aussagen über Tätigkeitszugänge und Aufgaben zu erhalten. Der Kern unserer Studie liegt in der Entschlüsselung von Qualifikationen und Arbeitsaufgaben. Ausgeklammert bleibt weitestgehend die Analyse des beruflichen Handelns in der BSA unter professionstheoretischen Perspektiven (vgl. z. B. Dewe und Stüwe 2016).

\section{Qualifikationen in der betrieblichen Sozialarbeit}

Fehlende Regelungen für das Berufsfeld bedingen eine Heterogenität der Qualifikationen des Personals in der BSA. Zwar gilt ein Hochschulstudium der Sozialarbeit/ Sozialpädagogik oft als Mindestqualifikation, allerdings sind auch Abschlüsse „vergleichbarer Fachrichtungen“ oder „anderer sozialwissenschaftlicher Fächer" möglich (vgl. Bremmer 2017b; Appelt 2013; bbs 2009). Zu letzteren gibt es jedoch keine Spezifikationen; gleiches gilt für die Forderung nach mehrjähriger berufspraktischer Erfahrung, deren Relevanz zwar vielfach ebenso betont wird (vgl. bbs 2009) wie die Absolvierung von Fort- und Weiterbildungen (vgl. Schröder 1961; Bremmer 2017b; bbs 2009), doch auch hier sind keine klaren inhaltlichen Schwerpunkte erkennbar: So wird z. B. im Report zum „European Seminar on Personnel Social Work " konstatiert, dass zunächst eine grundlegende Ausbildung ( „basic training“) in der Sozialen Arbeit benötigt wird, da die BSA auf den Prinzipien, Kenntnissen und Methoden der Sozialen Arbeit beruht (vgl. Schröder 1961). Forderungen nach darüber hinaus reichenden Weiterbildungen („supplementary training“) sind aber nicht spezifisch, sondern weisen eher vage Ausrichtungen entlang der Felder BWL, Arbeitspsychologie, Arbeitssoziologie, Arbeitsrecht und Personalmanagement auf (vgl. Schröder 1961). Ähnliche Aussagen finden sich bei Stoll (2013), die zu dem Schluss gelangt, dass sozialpädagogische Kenntnisse allein nicht ausreichen, um die Aufgaben der BSA adäquat bewältigen zu können und weitere Kenntnisse u. a. in BWL, Organisationsberatung und -entwicklung sowie Vertiefungen sozialpädagogischer Inhalte fordert.

Vor diesem Hintergrund haben wir im Rahmen unserer Studie untersucht, welche Qualifikationen seitens der Arbeitgeber gefordert (Qualifikationsanforderungen) und welche aus der Perspektive der BSA-MA für entsprechende Tätigkeiten benötigt werden (Qualifikationsvoraussetzungen). Aus dem Abgleich wurde das Qualifikationsprofil ermittelt (vgl. Nagel et al. 1999; Faulstich 1998).

In der Auswertung der Stellenausschreibungen zeigt sich bzgl. der formalen Qualifikation, dass 14 von 15 Arbeitgebern einen Studienabschluss der Sozialen Arbeit (Sozialarbeit, Sozialpädagogik) fordern. Von den Befragten haben sechs von sieben Personen ein entsprechendes Studium absolviert. Dieser Befund stärkt die Position, die vom Berufsfachverband (vgl. bbs 2009), von den Vereinten Nationen (vgl. Schröder 1961) sowie in einschlägigen Fachlexika (vgl. Bremmer 2017b; Appelt 2013) vertreten wird, nämlich dass es sich bei der BSA um ein Feld der Sozialen Arbeit handelt, in dem entsprechend ausgebildete Fachkräfte tätig sind. Ähnliches gilt für formale Weiterbildungen, welche in 13 der 15 Stellenanzeigen gefordert werden (überwiegend in der Beratung). Von den sieben Befragten hat jede Person mindestens eine formale Weiterbildung absolviert. Fünf Personen haben eine Weiterbildung im (systemischen) Coaching und zwei Personen eine in der Organisationsberatung absolviert. Die übrigen beziehen sich auf systemische Beratung, Case Management, Sucht- und Sozialtherapie, Business Moderation, Suchtberatung, Konfliktmanagement und Mediation sowie Sozialmanagement.

Weiterbildungen können wichtig sein, um mit Blick auf die Spezifika der BSA theoretische Kenntnisse zu vertiefen, wie zwei Aussagen aus dem Interviewmaterial verdeutlichen:

Und Suchtberatung ist ja einer unserer Schwerpunkte im Bereich der betrieblichen Sozialberatung. Und auch da Kenntnisse zu haben über Verläufe von Suchterkrankungen, Interventionsleitfäden im Umgang mit suchtmittelauffälligen Beschäftigten, das ist schon sehr hilfreich. Und alles, was Konfliktmanagement und Mediation anbelangt, auch das ist einer unserer Hauptschwerpunkte in der Sozialberatung. Und da tatsächlich auch nochmal so einen theori- ... theoretischen Überbau zu haben, das ist auch nochmal ganz wichtig. (BSA$M A G)$

Also (...) was ich tatsächlich eigentlich damals mit Erschrecken festgestellt habe, weiß nicht, wie das heute 
ist, mein Studium hat mich ... hat mich nicht wirklich sehr gut auf diesen Job vorbereitet, ne? Sondern für den Job war wichtig die ... die Ausbildung im ... in Beratungstechniken $z$ haben und einfach verschiedene Möglichkeiten da zu haben. Das ... das ist tatsächlich das A und O gewesen. [...] Nee, ich habe nicht ... ich habe nicht betriebliche Sozialarbeit studiert, aber ich bin ... habe ja den Abschluss als Sozialpädagogin im Endeffekt, ne, und da habe ich viele interessante Themen mitgebracht, aber ... aber es war jetzt keine konkrete Geschichte wie ... wie arbeite ich wirklich in der betrieblichen Sozialberatung damit? Weiß auch nicht, ob das sein muss, aber ... aber für mich war ... war wirklich wertvoll diese ... diese vertiefende Ausbildung in Beratungstechniken. (BSA-MA D)

Ferner zeigt sich, dass mehrjährige berufspraktische Erfahrung in Einsatzfeldern der Sozialen Arbeit ein wichtiges Kriterium darstellt, um Zugang zur BSA zu erhalten. In 11 von 15 Stellenanzeigen wird mehrjährige Berufserfahrung gefordert, in 12 Berufserfahrung mit konkretem Bezug zur Beratung. Von den Befragten konnte jede Person mehrjährige Berufserfahrung vorweisen, bevor sie ihre derzeitige Stelle in der BSA antraten. Der Schwerpunkt liegt in der Sozialen Arbeit (u. a. psychosoziale Beratungsstelle, sozialpädagogische Familienhilfe, Schulsozialarbeit, Betreuung psychisch kranker Menschen, Suchthilfe). Unabhängig von einzelnen Einsatzfeldern kann ein Fundus an Erfahrung Sicherheit geben, um Probleme der Klient_innen zu verstehen und passende Hilfemaßnahmen einzuleiten:

Die sind natürlich sehr hilfreich, weil ich hier ja ... ich sage mal ganz gemischte Klientel habe, die auch mit unterschiedlichen Sorgen und Nöten, (...) zu mir kommen dürfen und da hat man natürlich je mehr Erfahrung da ist und je mehr an Feldern, die schon mal beschnuppert wurden oder auf denen ich mich schon mal getummelt habe ... ist natürlich ... unterstützt natürlich hier. Ich sage mal, die Vorstellung dessen, was alles möglich ist, $j a$ ? Und auch die Wege ... welche Wege sind zu gehen und in welchen gesetzlichen Vorschriften und so weiter kann ich nachschlagen? Das ist natürlich dann schon mal ganz gut, wenn das so breit gefächert ist. (BSA$M A B)$

Ich habe mit jungen Erwachsenen gearbeitet. Ich habe mit Erwachsenen gearbeitet. Ich habe mit Rehabilitanden gearbeitet, also viel auch mit Leuten zu tun gehabt, die gesundheitliche Einschränkungen haben. Das ist was, was ich jetzt hier ja auch nutzen kann. Dadurch kenne ich das Hilfesystem sehr gut. (BSA-MA A)
Um die vielfältigen Anforderungen besser zu differenzieren, haben wir zudem eine Unterscheidung nach funktionalen und extrafunktionalen Qualifikationselementen vorgenommen (vgl. Dahrendorf 1956). Unter funktionalen Qualifikationselementen verstehen wir arbeitsplatzbezogene Kenntnisse, Fähigkeiten und Fertigkeiten, die benötigt werden, um bestimmte Arbeitsaufgaben einer Stelle zu erfüllen. Unter extrafunktionalen Qualifikationselementen verstehen wir hingegen (arbeitsplatz-)übergreifende Kenntnisse, Fähigkeiten und Fertigkeiten. Bei den funktionalen Qualifikationselementen sind es vor allem Kenntnisse im Sozialrecht, Kenntnisse der Suchtprävention und Suchtberatung, Kenntnisse und Fertigkeiten in der Beratung sowie Kenntnisse zur Organisation von Lernprozessen, die sowohl in den Stellenanzeigen gefordert als auch von den Befragten benannt werden. Auffällig ist, dass keine grundlegenden Fachkenntnisse in Bezug auf das Erwachsenenalter angeführt werden (z. B. Entwicklungsaufgaben, kritische Bewältigungskonstellationen und Übergänge, Lernen im Erwachsenenalter), obgleich Erwachsene das Kernklientel darstellen.

Ebenso wenig werden Arbeitsprinzipien und Methoden der Sozialen Arbeit thematisiert, obwohl diese mit Hinblick auf das Aufgabenprofil unverzichtbar sind. Während Letztere fester Bestandteil einschlägiger Studiengänge sind und vermutet werden kann, dass Arbeitgeber davon ausgehen, dass Bewerber/-innen diese Kenntnisse in einem einschlägigen Studiengang erworben haben, gilt dies nicht für fachliche Kenntnisse in Bezug auf den Umgang mit Erwachsenen.

Schließlich wird in den Ausschreibungen und in den Interviews eine Vielzahl von Erfordernissen benannt, die den extrafunktionalen Qualifikationselementen zugeordnet werden können, darunter Kenntnisse im Umgang mit Softwares, Englischkenntnisse, kommunikative Fähigkeiten, Organisationsfähigkeit, psychische Belastbarkeit, Flexibilität, äußeres Erscheinungsbild, Auftreten und Ausstrahlung, Lernbereitschaft, Teamund Kooperationsfähigkeiten, Empathie, Konfliktfähigkeit, Verhandlungsgeschick und Klientenorientierung. Durch den Abgleich der Qualifikationsanforderungen und -voraussetzungen lässt sich folgendes Profil für die betriebliche Sozialarbeit skizzieren (vgl. Tab. 1).

\section{Aufgaben in der betrieblichen Sozialarbeit}

In beiden Teiluntersuchungen zeigt sich, dass die psychosoziale Beratung, Begleitung und Unterstützung von Mitarbeiter_innen und Führungskräften die zentrale Aufgabe darstellt. In den Beratungsgesprächen werden Themen wie Konflikte im Privat- und/oder Berufsleben, berufliche Leistungsfähigkeit oder die eigene gesund- 
Tab. 1 Qualifikationsprofil für die BSA. (Quelle: Autoren)

\begin{tabular}{|c|c|}
\hline \multicolumn{2}{|c|}{ Qualifikationsprofil für die betriebliche Sozialarbeit } \\
\hline \multirow[t]{4}{*}{ Studium } & Soziale Arbeit (Sozialarbeit, Sozialpädagogik) \\
\hline & Psychologie \\
\hline & Pädagogik \\
\hline & Andere/vergleichbare Studiengänge \\
\hline \multirow[t]{5}{*}{ Formale Weiterbildungen } & (Systemische) Beratung \\
\hline & Konfliktmanagement und Mediation \\
\hline & Moderation \\
\hline & Systemisches Coaching \\
\hline & Case Management \\
\hline \multirow[t]{3}{*}{ Berufserfahrung } & Mehrjährige Berufserfahrung \\
\hline & Berufserfahrung in Feldern der Sozialen Arbeit und mit Bezug zur Beratung \\
\hline & Praxiserfahrung in Wirtschaft und/oder Verwaltung \\
\hline \multirow[t]{4}{*}{ Funktionale Qualifikationselemente } & Kenntnisse im Sozialrecht \\
\hline & Kenntnisse der Suchtprävention und Suchtberatung \\
\hline & Kenntnisse und Fertigkeiten in der Beratung \\
\hline & Kenntnisse zur Organisation von Lernprozessen \\
\hline \multirow[t]{13}{*}{ Extrafunktionale Qualifikationselemente } & Kenntnisse im Umgang mit PC-Softwares (vor allem MS Office-Anwendungen) \\
\hline & Fremdsprachenkenntnisse (Englisch) \\
\hline & Kommunikative Fähigkeiten \\
\hline & Organisationsfähigkeit \\
\hline & Psychische Belastbarkeit \\
\hline & Flexibilität \\
\hline & Äußeres Erscheinungsbild, Auftreten und Ausstrahlung \\
\hline & Lernbereitschaft \\
\hline & Team- und Kooperationsfähigkeiten \\
\hline & Empathie \\
\hline & Konfliktfähigkeit \\
\hline & Verhandlungsgeschick \\
\hline & Klientenorientierung \\
\hline
\end{tabular}

heitliche Verfassung thematisiert. Entgegen bisheriger Forschungsbefunde (z. B. Baumgartner und Sommerfeld 2016) spielt die Suchtberatung eine eher untergeordnete Rolle. Des Weiteren gehören zu dieser Aufgabe die Koordination weiterführender Hilfsangebote, die Unterstützung bei sozialorganisatorischen Angelegenheiten (z. B. Ausfüllen von Reha-, Renten- und Schwerbehindertenanträgen, Beantragung einer Pflegestufe) sowie mitunter auch das Führen von Mutterschutzgesprächen. Das Konfliktmanagement umfasst interventive Tätigkeiten (z. B. Durchführung von Konfliktklärungsgesprächen) und präventive Tätigkeiten (z. B. vorbeugende Konfliktberatung in Organisationsentwicklungsprozessen). Es wird von einem Großteil der Befragten als eine Hauptaufgabe der BSA angesehen, was sich mit bisherigen Forschungsbefunden deckt (vgl. Baumgartner und Sommerfeld 2016).
Unter Personalentwicklung sind bildende und fördernde Maßnahmen subsumiert. In unterschiedlichen Lehr-Lern-Formaten wie Seminaren, Workshops und Vorträgen bietet die BSA die Auseinandersetzung mit unterschiedlichen Themen an, welche sich in den Bereichen gesundheitliche Bildung (z. B. Umgang mit Stress und Belastungen) und berufliche Weiterbildung ( $\mathrm{z}$. B. Führen von Mitarbeitergesprächen) verorten lassen. Für die Zielgruppe der Führungskräfte stellt das Coaching eine Kerntätigkeit dar (vgl. auch Baumgartner und Sommerfeld 2016; Stoll 2013).

Im Rahmen des betrieblichen Gesundheitsmanagements (BGM) ist die Projektarbeit von besonderer Bedeutung. Von unseren Befragten sind zwei Personen für Projektleitung und -steuerung verantwortlich. Andere sind als Projektmitarbeiter_in tätig und übernehmen in diesem Rahmen spezifische Aufgaben wie konzeptionel- 
le Arbeit, die Erstellung von Statistiken oder Netzwerkarbeit. Im Zusammenhang mit dem BGM ist zudem das betriebliche Eingliederungsmanagement (BEM) zu sehen, das ebenfalls als Kerntätigkeit eingeschätzt wird. Auch hier ist eine Differenzierung vorzunehmen zwischen Sozialberatungsstellen, die den Prozess federführend steuern, und anderen Stellen, die bei der Bearbeitung von Einzelfällen unterstützend tätig sind.

Eine weitere Aufgabe ist die Entwicklung von Angeboten der BSA: Sie beinhaltet den Ausbau der Sozialberatungsstelle an anderen Standorten und die Weiterentwicklung der Angebote. So schilderten mehrere Befragte, dass durch die zunehmende Digitalisierung neue Angebotsformen (z. B. Online-Beratung, E-Learning-Formate) nachgefragt und teilweise schon umgesetzt werden.

Um Mitarbeiter_innen, Führungskräfte und ggf. Angehörige auf das Leistungsspektrum der Sozialberatungsstelle aufmerksam zu machen, spielt die Öffentlichkeitsarbeit eine wichtige Rolle (z. B. Durchführung von Informationsveranstaltungen oder Gestaltung des Internetauftritts).

Im Aufgabenschwerpunkt Netzwerkmanagement sind sowohl der Aufbau und die Pflege von Kontakten zu externen Einrichtungen (z. B. Kliniken, Beratungsstellen) als auch die Zusammenarbeit mit betriebsinternen Personen und/oder Bereichen (z. B. in Gremien, Arbeitskreisen) inbegriffen.

Nicht zuletzt umfasst das Aufgabenprofil in der BSA auch Administratives, wie etwa das Anfertigen von Dokumentationen und Berichten, Aktenführung und Recherchearbeit.

\section{Fazit}

In unserer Untersuchung konnten wir eine Reihe von Forschungsbefunden zu Qualifikationen und Aufgaben in der BSA bestätigen und konkretisieren, so z. B. Qualifikationserfordernisse bzgl. der Studienabschlüsse, Weiterbildungen und Berufserfahrungen. Wir haben zudem gezeigt, dass neben „klassischen Aufgaben“, wie etwa die psychosoziale Einzelfallarbeit, auch Aufgaben wie internes und externes Netzwerkmanagement oder die stetige Weiterentwicklung der Angebote eine wichtige Rolle spielen. Zudem hat sich gezeigt, dass die BSA als Tätigkeitsfeld zwar keiner gesetzlichen Verankerung unterliegt, aber BSA-MA Tätigkeiten übernehmen, für die sie detaillierte rechtliche Kenntnisse benötigen (z. B. BEM, psychische Gefährdungsbeurteilung). Das Aufgabenprofil reicht teils deutlich über typische sozialarbeiterische/-pädagogische Tätigkeiten hinaus und weist Schnittstellen zu Elementen des (Sozial-)Managements auf. Die Schnittstellen werden besonders bei Tätigkeiten im Bereich Personalentwicklung, Angebotsentwicklung, Öffentlichkeitsarbeit und Netzwerkmanagement sichtbar und akzentuieren den Bedarf an interdisziplinären Kenntnissen, Fähigkeiten und Fertigkeiten.

\section{ส้อง}

Funding. Open Access funding provided by Projekt DEAL.

Open Access. Dieser Artikel wird unter der Creative Commons Namensnennung 4.0 International Lizenz veröffentlicht, welche die Nutzung, Vervielfältigung, Bearbeitung, Verbreitung und Wiedergabe in jeglichem Medium und Format erlaubt, sofern Sie den/die ursprünglichen Autor(en) und die Quelle ordnungsgemäß nennen, einen Link zur Creative Commons Lizenz beifügen und angeben, ob Änderungen vorgenommen wurden.

Die in diesem Artikel enthaltenen Bilder und sonstiges Drittmaterial unterliegen ebenfalls der genannten Creative Commons Lizenz, sofern sich aus der Abbildungslegende nichts anderes ergibt. Sofern das betreffende Material nicht unter der genannten Creative Commons Lizenz steht und die betreffende Handlung nicht nach gesetzlichen Vorschriften erlaubt ist, ist für die oben aufgeführten Weiterverwendungen des Materials die Einwilligung des jeweiligen Rechteinhabers einzuholen.

Weitere Details zur Lizenz entnehmen Sie bitte der Lizenzinformation auf http://creativecommons.org/licenses/by/4.0/deed.de

\section{Literatur}

Appelt, H.-J. (2013). Betriebliche Sozialarbeit. In D. Kreft \& I. Mielenz (Hrsg.), Wörterbuch Soziale Arbeit. Aufgaben, Praxisfelder, Begriffe und Methoden der Sozialarbeit und Sozialpädagogik (7. Aufl. S. 176-177). Weinheim/Basel: Beltz Juventa.

Baumgartner, E., \& Sommerfeld, P. (2016). Betriebliche Soziale Arbeit. Empirische Analyse und theoretische Verortung. Wiesbaden: Springer.

Blandow, J. (1993). Betriebliche Sozialarbeit - Von der Fabrikpflege auf dem Weg wohin? Theorie und Praxis der Sozialen Arbeit, 44(8), 312-319.

Bremmer, M. (2017a). 100 Jahre betriebliche Sozialarbeit - Entwicklung, Geschichte und Wandel der Betriebssozialarbeit. In S. Klein \& H.-J. Appelt (Hrsg.), Praxishandbuch betriebliche Sozialarbeit. Prävention und Interventionen in modernen Unternehmen (6. Aufl. S. 9-18). Kröning: Asanger.

Bremmer, M. (2017b). Betriebliche Sozialarbeit. In Deutscher Verein für öffentliche und private Fürsorge e. V. (Hrsg.), Fachlexikon der Sozialen Arbeit (8. Aufl. S. 110-111). Baden-Baden: Nomos.

Bundesfachverband Betriebliche Sozialarbeit e. V. [bbs] (2009). Rabmenkonzeption für das Arbeitsfeld Betriebliche Sozialarbeit. Tübingen: Bundesfachverband Betriebliche Sozialarbeit e. V..

Dahrendorf, R. (1956). Industrielle Fertigkeiten und soziale Schichtung. Kölner Zeitschrift für Soziologie und Sozialpsychologie, 8, 540-568.

Dewe, B., \& Stüwe, G. (2016). Basiswissen Profession. Zur Aktualität und kritischen Substanz des Professionalisierungskonzeptes für die Soziale Arbeit. Weinheim/Basel: Beltz Juventa.

Faulstich, P. (1998). Strategien der betrieblichen Weiterbildung. Kompetenz und Organisation. München: Vahlen.

Flick, U. (2008). Triangulation. Eine Einführung (2. Aufl.). Wiesbaden: VS/GWV.

Klein, S., \& Appelt, H.-J. (2017). Einführung der Herausgeber. In S. Klein \& H.-J. Appelt (Hrsg.), Praxishandbuch betriebliche Sozialarbeit. Prävention und Interventionen in modernen Unternehmen (6. Aufl. S. 5-7). Kröning: Asanger.

Mayring, P. (2016). Einführung in die qualitative Sozialforschung. Eine Anleitung zu qualitativem Denken (6. Aufl.). Weinheim/Basel: Beltz. 
Nagel, R., Oswald, M., \& Wimmer, R. (1999). Das Mitarbeitergespräch als Führungsinstrument. Stuttgart: Klett-Cotta.

Psychische Gesundheit in der Arbeitswelt [psyGA] (2017). Kein Stress mit dem Stress. Ein Leitfaden zur Auswabl von Angeboten der Mitarbeiterberatung. Berlin: Initiative Neue Qualität der Arbeit.

Schröder, M. (1961). Summary of conclusions of the seminar. In United Nations (Hrsg.), European seminar on personnel social work. Report (S. 7-21). Geneva: United Nations.

Stoll, B. (2013). Betriebliche Sozialarbeit. Aufgaben und Bedeutung. Praktische Umsetzung (2. Aufl.). Regensburg: Walhalla.

Witzel, A. (1985). Das problemzentrierte Interview. In G. Jüttemann (Hrsg.), Qualitative Forschung in der Psychologie: Grundfragen, Verfahrensweisen, Anwendungsfelder (S. 227-255). Weinheim/Basel: Beltz.

\section{Hier steht eine Anzeige.}

\section{Springer}

